Pesq. Vet. Bras. 37(6):593-597, junho 2017

DOI: $10.1590 / \mathrm{S} 0100-736 \mathrm{X} 2017000600010$

\title{
Pesquisa de hemoglobinopatias em cães da região metropolitana do Rio de Janeiro portadores de anemia crônica ${ }^{1}$
}

\author{
Gabriel Bobany de Queiroz ${ }^{2 *}$, Sérgio Lisboa Machado³, Helena Keiko Toma ${ }^{4}$, Nayro \\ Xavier Alencar ${ }^{5}$, Daniel Barros Macieira ${ }^{6}$ e Nadia Regina Pereira Almosny ${ }^{7}$
}

\begin{abstract}
Queiroz G.B., Machado S.L., Toma H.K., Alencar N.X., Macieira D.B. \& Almosny N.R.P. 2017. [Search for hemoglobinopathies in dogs with chronic anemia in the metropolitan region of Rio de Janeiro.] Pesquisa de hemoglobinopatias em cães da região metropolitana do Rio de Janeiro portadores de anemia crônica. Pesquisa Veterinária Brasileira 37(6):593-597. Departamento de Clínica e Reprodução Animal, Faculdade de Veterinária, Universidade Federal Fluminense, Rua Vital Brazil Filho 64, Vital Brazil, Niterói, RJ 24230-340, Brazil.E-mail: bobany@gmail.com

Thalassemias and hemoglobinopathies are hereditary conditions found in humans throughout the world. In veterinary medicine, hemoglobin polymorphism has been studied in production animals, but there are no reports of hemoglobinopathies in dogs, and studies involving hemoglobin polymorphism in this species are scarce. In order to search for hemoglobin variants in dogs, blood samples were collected from 202 dogs of various breeds, being 130 patients with chronic anemia (Experimental Group) and 72 clinically healthy animals (Control Group). These samples were subjected to alkaline electrophoresis of hemoglobin, which permitted separation and quantification of hemoglobin fractions by densitometry, and then subjected to hemoglobin electrophoresis in an acid medium, a technique used in human medicine for the separation of variant fractions of hemoglobin that do not differentiate in an alkaline medium. The erythrogram and RBC indices were obtained concurrently. The methods demonstrated that $\mathrm{HbA}$ is the major component of canine hemoglobin, and a small amount of $\mathrm{HbA} 2$ can be detected in some of the evaluated animals, and most dogs showed only $\mathrm{HbA}$ in its composition. It was concluded that the presence or absence of HbA2 does not interfere with RBC indices of evaluated animals, and the comparison between the hemoglobin of Experimental and Control groups showed no differences in fractions distribution between them, and there was no hemoglobin variants in evaluated canines.
\end{abstract}

INDEX TERMS: Hemoglobinopathies, electrophoresis, hemoglobin polymoprphism, dogs.

${ }^{1}$ Recebido em 15 de janeiro de 2016.

Aceito para publicação em 18 de julho de 2016

${ }^{2}$ Departamento de Clínica e Reprodução Animal, Faculdade de Veterinária, Universidade Federal Fluminense (UFF), Rua Vital Brazil Filho 64, Vital Brazil, Niterói, RJ 24230-340, Brasil. *Autor para correspondência: bobany@gmail.com

${ }^{3}$ Faculdade de Farmácia, Universidade Federal do Rio de Janeiro (UFRJ), Av. Carlos Chagas Filho 373, Prédio do CCS, Bloco A, $2^{\circ}$ andar sala 53, Ilha do Fundão, Rio de Janeiro, RJ 21944970, Brasil.

${ }^{4}$ Faculdade de Farmácia, UFRJ, Av. Carlos Chagas Filho 373, Prédio do CCS, Bloco A, 2 o andar sala 13, Ilha do Fundão, Rio de Janeiro, RJ 21944970.

${ }^{5}$ Departamento de Clínica e Reprodução Animal, Faculdade de Veterinária, UFF, Rua Vital Brazil Filho 64, Vital Brazil, Niterói, RJ 24230-340, Brasil.

${ }^{6}$ Departamento de Clínica e Reprodução Animal, Faculdade de Veterinária, UFF, Rua Vital Brazil Filho 64, Vital Brazil, Niterói, RJ 24230-340, Brasil.

${ }^{7}$ Departamento de Clínica e Reprodução Animal, Faculdade de Veterinária, UFF, Rua Vital Brazil Filho 64, Vital Brazil, Niterói, RJ 24230-340, Brasil.
RESUMO.- Talassemias e hemoglobinopatias são condições hereditárias encontradas em humanos de todo o mundo. Em medicina veterinária, o polimorfismo de hemoglobinas tem sido estudado em animais de produção, mas não existem relatos de hemoglobinopatias em cães, e os estudos envolvendo o polimorfismo de hemoglobinas nesta espécie são escassos. Com o objetivo de pesquisar variantes da hemoglobina em cães, foram coletadas amostras de sangue de 202 caninos de variadas raças, sendo 130 portadores de anemia crônica (Grupo Experimental) e 72 animais clinicamente saudáveis (Grupo Controle). Estas amostras foram submetidas à eletroforese alcalina de hemoglobinas, que permitiu a separação e quantificação das frações de hemoglobina por densitometria, e posteriormente submetidas à eletroforese de hemoglobinas em meio ácido, técnica utili- 
zada em medicina humana para a separação de frações de hemoglobinas variantes que não se diferenciam em meio alcalino. 0 eritrograma e índices hematimétricos foram obtidos concomitantemente. Os métodos utilizados demonstraram que a $\mathrm{HbA}$ é o maior componente da hemoglobina canina, e que uma pequena quantidade de $\mathrm{HbA} 2$ pode ser detectada em uma parcela dos animais avaliados, sendo que a maioria dos caninos apresentava exclusivamente $\mathrm{HbA}$ em sua composição. Concluiu-se que a presença ou ausência de $\mathrm{HbA2}$ não interfere nos índices hematimétricos dos animais avaliados, e que quando comparadas as hemoglobinas dos grupos Experimental e Controle, não são observadas diferenças na distribuição das frações destas, além de não serem observadas hemoglobinas variantes nos caninos avaliados.

TERMOS DE INDEXAÇÃO: Hemoglobinopatias, eletroforese, polimorfismo de hemoglobinas, caninos.

\section{INTRODUÇÃO}

Talassemias e hemoglobinopatias são condições hereditárias encontradas em seres humanos de todo o mundo (Wagner et al. 2005, Srivorakun et al. 2011, Lin et al. 2012). As anemias hereditárias são, das doenças geneticamente determinadas, as mais comuns e compreendem um grupo de condições de considerável complexidade (Leoneli et al. 2000).

As hemoglobinopatias são um assunto de interesse crescente entre as autoridades de saúde e pesquisadores em medicina humana no Brasil, uma vez que diferentes estudos apontam que estes distúrbios genéticos são um problema de saúde pública nacional, e desde 2001 o Ministério da Saúde tornou obrigatória a pesquisa de hemoglobinopatias em recém-nascidos, usando o teste de Guthrie (Seixas et al. 2008).

Em medicina veterinária, o polimorfismo da hemoglobina tem sido estudado mais intensamente em animais de produção. 0 tipo de hemoglobina pode apresentar relação com a resistência a parasitos, intensidade de resposta imune e relação com eficiência reprodutiva e produção de lã e leite, e é empregado na identificação de indivíduos e de parentesco, é um dado útil, na seleção de animais com aspectos genéticos ligados a interesse produtivo (Lacerda \& Soto-Blanco 2006, Luz et al. 2010, Ayres et al. 2011).

Diversos polimorfismos de hemoglobinas já foram descritos em espécies animais, como bovinos, bubalinos, ovinos, caprinos e felinos (Ramos et al. 1997, Regis 2002, Ayres et al. 2005, Lacerda \& Soto-Blanco 2006, Esharatkhah et al. 2007, Barca Júnior et al. 2008, Ayres et al. 2009, Al-Samarrae et al. 2010, Luz et al. 2010, Ayres et al. 2011, Rego et al. 2011, Atyabi et al. 2012, Egena \& Alao 2014, Pal \& Mummed 2014, Yakubu et al. 2014). No entanto, não há na literatura relatos de hemoglobinopatias envolvendo cães, e os estudos sobre o polimorfismo das hemoglobinas nesta espécie são escassos.

Sendo assim, os objetivos principais deste estudo foram comparar os tipos de hemoglobina de cães que apresentem anemias crônicas com os clinicamente saudáveis pelos métodos da eletroforese alcalina e ácida, objetivando-se identificar diferenças na estrutura destas hemoglobinas, e evidenciando possíveis hemoglobinopatias nestes pacientes. Secundariamente, o estudo visou comparar índices hematimétricos de cães que apresentassem tipos diferentes de hemoglobina em sua composição, a fim de detectar se as diferentes concentrações de tipos de hemoglobina influenciam o comportamento hematológico destes cães.

\section{MATERIAL E MÉTODOS}

O estudo foi autorizado pelo Comitê de Ética no Uso de Animais da Universidade Federal Fluminense sob o protocolo 724.

Foram avaliados cães da região metropolitana do Rio de Janeiro atendidos no Hospital Veterinário Universitário Professor Firmino Mársico Filho da Universidade Federal Fluminense (Huvet/UFF) entre o período de março de 2012 a junho de 2015. Os animais foram separados em dois grupos:

i) Grupo Experimental: foram selecionados, a partir de registros de pacientes do Hospital Huvet/UFF, 130 animais da espécie canina, independente de sexo ou raça, com idade acima de 1 ano de idade, que apresentassem hematócrito abaixo de 37\% por, no mínimo, 15 dias e, no máximo, 30 dias sem determinação da causa da anemia, com ou sem sintomatologia clínica de doença e independente da terapêutica adotada, desde que a mesma não interferisse no hematócrito. Foram: excluídos do estudo os animais que apresentassem anemia por doença renal crônica, com valores de creatinina sérica acima de 1,5g/dL; animais com diagnóstico de neoplasia de qualquer origem; animais com leucocitose; animais com diagnóstico de alguma hemoparasitose por qualquer agente etiológico.

ii) Grupo Controle: foram selecionados, também a partir de registros de pacientes do Huvet/UFF, 72 animais da espécie canina, independente de sexo ou raça, com idade acima de 1 ano de idade, que apresentassem hematócrito acima de $37 \%$ e clinicamente sadios. 0 hemograma foi realizado de forma automatizada pelo Analisador Hematológico Veterinário pocH-100iV Diff (Sysmex ${ }^{\circledR}$ ) para a obtenção do eritrograma. A eletroforese alcalina de hemoglobinas foi realizada utilizando kits da marca CELMGEL ${ }^{\circledR}$.

0 hemolisado foi obtido através da técnica descrita por Naoum (1987) e submetido à eletroforese em meio alcalino (pH 9,5) e posteriormente corado pelo Ponceau, seguindo as orientações do fabricante $\left(\mathrm{CELM}^{\circledR}\right)$, e submetido à leitura densitométrica pelo Software para Densitometria por Scanner SDS-60. 0 hemolisado também foi submetido à eletroforese em meio ácido $(\mathrm{pH} 6,2)$ para separação eletroforética de bandas de hemoglobina que porventura migrassem em padrão semelhante no meio alcalino.

Como controle para validação das técnicas, foi utilizado sangue humano sem hemoglobinopatias, visto que as técnicas já são validadas para separação eletroforética da hemoglobina humana em frações de Hemoglobina A, Hemoglobina Fetal e Hemoglobina A2, além de hemoglobinas variantes.

Os dados obtidos foram analisados pelo teste estatístico de Mann-Whitney para testar a hipótese de que havia diferença entre as medianas das concentrações das diferentes frações de hemoglobinas entre os grupos, bem como para avaliar se havia diferença entre os valores do eritrograma em animais com diferentes morfologias das frações de hemoglobina.

\section{RESULTADOS E DISCUSSÃO}

0 valor médio do hematócrito dos 202 animais avaliados foi de $35,72 \%$, e a concentração de hemoglobina média foi de $11,74 \mathrm{~g} / \mathrm{dL}$. Os índices hematimétricos encontram-se no Quadro 1. 
Quadro 1. Médias e desvios padrão dos valores hematológicos dos animais avaliados da região metropolitana do Rio de Janeiro e valores de referência para as variáveis do hemograma

\begin{tabular}{|c|c|c|c|}
\hline Parâmetro & Média & DP & VR† \\
\hline $\mathrm{He}(\mathrm{x} 106 / \mu \mathrm{L})$ & 5,00 & 1,66 & $5,5-8,5$ \\
\hline $\mathrm{Hb}(\mathrm{g} / \mathrm{dL})$ & 11,74 & 3,86 & $12-18$ \\
\hline Ht (\%) & 35,72 & 11,22 & $37-55$ \\
\hline VGM (fL) & 72,34 & 6,70 & $60-77$ \\
\hline CHGM (\%) & 32,78 & 2,18 & $32-36$ \\
\hline HbA (\%) & 99,53 & 0,67 & * \\
\hline HbA2 (\%) & 0,47 & 0,67 & * \\
\hline
\end{tabular}

$\overline{\mathrm{DP}}=$ Desvio Padrão; VR = Valor de Referência; $\mathrm{He}=$ Hematimetria; $\mathrm{Hb}=$ Hemoglobina; Ht = Hematócrito; VGM = Volume Globular Médio; CHGM = Concentração de Hemoglobina Globular Média; $\mathrm{HbA}=$ Hemoglobina A; $\mathrm{HbA} 2$ = Hemoglobina $\mathrm{A} 2{ }^{*}{ }^{*}=$ Valor de referência não definido; $†$ (Jain 1993).

A partir do grupo controle foram estabelecidos valores de referência para o perfil eletroforético da hemoglobina em meio alcalino e ácido, tendo em vista que a eletroforese ácida pode diferenciar frações da hemoglobina que se movimentam de maneira semelhante à eletroforese alcalina.

A maior parte da concentração de hemoglobina nas amostras de sangue de ambos os grupos avaliados foi de $\mathrm{HbA}$. A HbA canina migrou mais lentamente que a $\mathrm{HbA} \mathrm{hu-}$ mana, porém mais rapidamente que a $\mathrm{HbA} 2$ canina, tanto em meio alcalino quanto em meio ácido (Fig.1). Não foram encontradas bandas com outro padrão de migração em nenhuma das duas técnicas.

A partir deste resultado, os animais foram separados em um grupo que apresentou apenas $\mathrm{HbA}$ e outro com aqueles que apresentaram predomínio de $\mathrm{HbA}$ com uma pequena fração de $\mathrm{HbA2}$, afim de se comparar os índices hematimétricos entre os dois grupos.

Não foi observada diferença macroscópica no padrão eletroforético macroscópico entre os animais tanto em meio alcalino quanto em meio ácido (Fig.2).

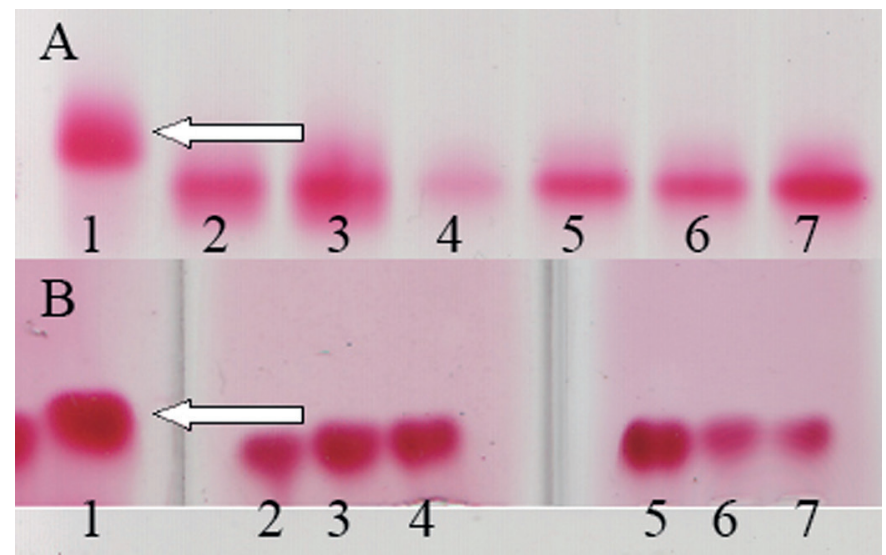

Fig.1. (A) Padrão eletroforético da hemoglobina em meio alcalino. Hemoglobina humana (1); Hemoglobina canina (2-7). A hemoglobina humana (seta) migrou mais rapidamente que a hemoglobina canina. (B) Padrão eletroforético da hemoglobina em meio ácido. Hemoglobina humana (1); Hemoglobina canina (2-7). A hemoglobina humana (seta) migrou mais rapidamente que a hemoglobina canina.
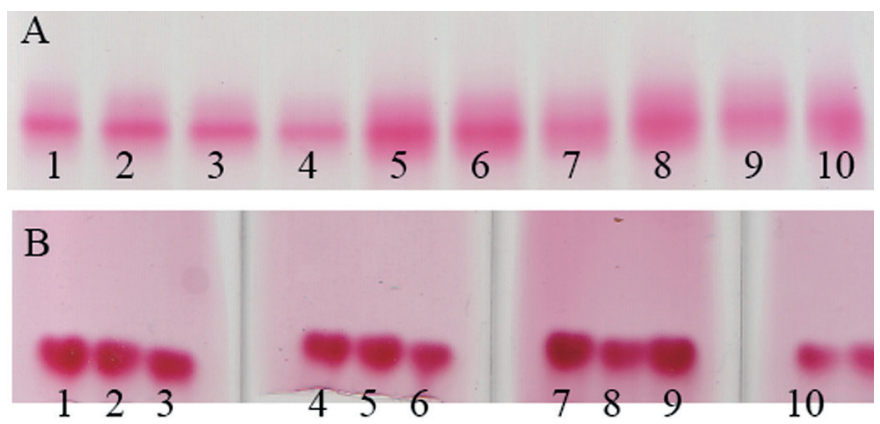

Fig.2. (A) Padrão eletroforético da hemoglobina canina em meio alcalino. (B) Padrão eletroforético da hemoglobina canina em meio ácido. 0 padrão de migração em ambos os métodos apresenta apenas uma banda visível com padrão de migração semelhante. Amostras do Grupo Controle (1-4); Animais do Grupo Experimental (5-10).

Quadro 2. Distribuição das medidas estatísticas de hematócrito e hemoglobina (A e A2) em amostras de sangue canino com anemias crônicas (Grupo Experimental) e animais saudáveis (Grupo Controle) coletadas de cães da região metropolitana do Rio de Janeiro

\begin{tabular}{|c|c|c|c|c|c|}
\hline \multirow[b]{2}{*}{ Parâmetro } & \multicolumn{2}{|c|}{$\begin{array}{l}\text { Grupo experimental } \\
(\mathrm{n}=130 / 202)\end{array}$} & \multicolumn{2}{|c|}{$\begin{array}{c}\text { Grupo controle } \\
(\mathrm{n}=72 / 202)\end{array}$} & \multirow[b]{2}{*}{$\mathrm{p}^{*}$} \\
\hline & Média & DP & Média & DP & \\
\hline Ht (\%) & 28,57 & 5,95 & 48,63 & 5,43 & 0,513 \\
\hline $\mathrm{Hb}(\mathrm{G} / \mathrm{dL})$ & 9,27 & 2,06 & 16,22 & 1,71 & 0,780 \\
\hline $\mathrm{HbA}(\%)$ & 99,52 & 0,66 & 99,54 & 0,71 & 0,727 \\
\hline $\mathrm{HbA2}(\%)$ & 0,48 & 0,66 & 0,46 & 0,71 & 0,727 \\
\hline
\end{tabular}

$\overline{\mathrm{DP}}=$ Desvio Padrão; $\mathrm{Ht}=$ Hematócrito; $\mathrm{Hb}=$ Hemoglobina; HbA = Hemoglobina A; HbA2 = Hemoglobina A2. * Obtido através do teste de Mann-Whitney.

Quadro 3. Distribuição dos índices hematimétricos em cães com apenas HbA em sua composição de hemoglobina e cães com pequena presença de $\mathrm{HbA2}$ em sua composição de hemoglobina em amostras coletadas de cães da região metropolitana do Rio de Janeiro

\begin{tabular}{|c|c|c|c|c|c|}
\hline \multirow[b]{3}{*}{ Parâmetro } & \multicolumn{4}{|c|}{ Todos os pacientes } & \multirow[b]{3}{*}{$\mathrm{p}^{*}$} \\
\hline & \multicolumn{2}{|c|}{$\begin{array}{c}100 \% \mathrm{HbA} \\
(\mathrm{n}=113 / 202)\end{array}$} & \multicolumn{2}{|c|}{$\begin{array}{l}\mathrm{HbA}+\mathrm{HbA2} \\
(\mathrm{n}=89 / 202)\end{array}$} & \\
\hline & Média & DP & Média & DP & \\
\hline He $(106 / \mathrm{uL})$ & 5,01 & 1,73 & 4,99 & 1,59 & 0,882 \\
\hline $\mathrm{Hb}(\mathrm{G} / \mathrm{dL})$ & 11,76 & 3,97 & 11,73 & 3,74 & 0,839 \\
\hline Ht $(\%)$ & 35,84 & 11,69 & 35,57 & 10,65 & 0,991 \\
\hline VGM (fL) & 72,37 & 6,48 & 72,30 & 7,02 & 0,796 \\
\hline CHGM (\%) & 32,77 & 2,35 & 32,80 & 1,94 & 0,800 \\
\hline \multicolumn{6}{|c|}{ Pacientes com anemia crônica } \\
\hline & \multicolumn{2}{|c|}{$\begin{array}{c}100 \% \mathrm{HbA} \\
(\mathrm{n}=113 / 202)\end{array}$} & \multicolumn{2}{|c|}{$\begin{array}{l}\mathrm{HbA}+\mathrm{HbA} 2 \\
(\mathrm{n}=89 / 202)\end{array}$} & \\
\hline Parâmetro & Média & DP & Média & DP & $\mathrm{p}^{*}$ \\
\hline He $(106 / u L)$ & 3,90 & 0,93 & 4,05 & 0,98 & 0,307 \\
\hline $\mathrm{Hb}(\mathrm{G} / \mathrm{dL})$ & 9,15 & 2,04 & 9,41 & 2,08 & 0,395 \\
\hline Ht $(\%)$ & 28,26 & 6,11 & 28,96 & 5,78 & 0,555 \\
\hline VGM (fL) & 73,30 & 7,50 & 72,87 & 8,31 & 0,970 \\
\hline CHGM (\%) & 32,48 & 2,79 & 32,39 & 2,24 & 0,466 \\
\hline
\end{tabular}

DP = Desvio Padrão; He = Hematimetria; Hb = Hemoglobina; Ht = Hematócrito; VGM = Volume Globular Médio; CHGM = Concentração de Hemoglobina Globular Média; HbA = Hemoglobina A; HbA2 = Hemoglobina A2. * Obtido através do teste de Mann-Whitney. 
Após análise densitométrica, assim como observado por Atyabi et al. (2012), a maior parte dos componentes de hemoglobina se localizaram no final anodo aproximadamente na mesma linha, sendo classificado como $\mathrm{HbA}$, mas em alguns casos, valores baixos de HbA2 também foram observados na avaliação densitométrica com um padrão mais lento de migração. Desta forma, pôde-se dividir em percentuais cada fração de hemoglobina presente nos animais.

A distribuição média da HbA de todos os animais envolvidos no estudo foi de 99,53\%, com uma concentração média de $\mathrm{HbA} 2$ de $0,47 \%$. 0 valor mínimo de $\mathrm{HbA}$ encontrado foi de $96,70 \%$ em um animal que apresentava $3,30 \%$ de $\mathrm{HbA2}$, e alguns animais apresentavam 100\% de HbA. Entre os animais portadores de anemias crônicas, a concentração média de $\mathrm{HbA}$ foi de 99,52\%, com uma distribuição média de $\mathrm{HbA} 2$ de $0,48 \%$. No Quadro 2 observa-se a distribuição das medidas estatísticas de hematócrito, hemoglobina e frações de hemoglobina (A e A2) em amostras de cães do Grupo Experimental e do Grupo Controle. Em cães saudáveis, as concentrações de $\mathrm{HbA}$ e $\mathrm{HbA} 2$ médias foram de $99,54 \%$ e $0,46 \%$, respectivamente.

Os animais anêmicos que possuíam exclusivamente HbA em sua composição apresentaram valor de hematócrito médio de $28,26 \%$ e 9,15g/dL, em comparação às concentrações respectivas de $28,96 \%$ e 9,41g/dL dos animais anêmicos que apresentavam em sua composição uma pequena fração de HbA2. A diferença não foi significativa, tendo sido observado, assim, que a composição de hemoglobina não interfere nos índices hematimétricos No quadro 3 estão apresentadas as concentrações médias de hematócrito e concentração de hemoglobina dos animais que possuíam apenas $\mathrm{HbA}$ em sua composição e aqueles que possuíam $\mathrm{HbA}$ e HbA2 em menor concentração.

Os resultados da separação eletroforética em meio ácido são apresentados qualitativamente para cada fração de hemoglobina observada como Presente ou Ausente. Para $100 \%$ dos animais avaliados neste estudo, foi observada presença de uma única banda relativa à $\mathrm{HbA}$. Em nenhum animal foi observado padrão de migração mais lento ou mais rápido de bandas de hemoglobina, que poderiam indicar hemoglobinas variantes.

Os resultados obtidos neste estudo são semelhantes aos relatados por Seal (1969), Naik et al. (1971) e Atyabi et al. (2012), que apenas encontraram frações de $\mathrm{HbA}$ em estudos por eletroforese alcalina em cães. No entanto, diferentemente de Atyabi et al. (2012), não foi observada a presença de hemoglobina fetal em meio alcalino. No atual estudo foram avaliados os perfis eletroforéticos apenas de animais em idade reprodutiva, enquanto estes autores avaliaram animais a partir dos quatro meses de idade. Estes autores, não observaram diferenças nas concentrações de hemoglobinas. Eles, no entanto, relatam a presença de hemoglobina fetal, embora não tenham especificado, exatamente, em qual idade média e raças esta hemoglobina foi observada.

Não foram encontrados outros tipos de hemoglobina ou outros genótipos através da eletroforese alcalina, como $\mathrm{HbB}$ e $\mathrm{HbAB}$, como encontrados por Tanabe et al. 1978 no Japão em 1978, que avaliou apenas cães de raças japone- sas nativas e cães de raças europeias. Esta diferença de genótipos de hemoglobina pode estar associada à seleção de raças mais regional e apurada. No entanto, além de Tanabe et al. (1978), em nenhum outro trabalho foi descrito um polimorfismo com dois tipos de hemoglobina em cães (Seal 1969, Kitchen \& Brett 1974, Atyabi et al. 2012).

Não foi observada diferença significativa entre as concentrações de $\mathrm{HbA}$ e $\mathrm{HbA} 2$ entre os grupos Experimental e Controle $(p=0,727)$. Não houve diferença na comparação da $\mathrm{Hb}$ entre animais anêmicos e não anêmicos. Não foram encontrados, entretanto, na literatura estudos comparando as concentrações dos dois tipos de hemoglobina entre animais saudáveis e animais com anemias crônicas. Atyabi et al. (2012) realizaram a avaliação das concentrações de hemoglobina A e A2 em animais saudáveis, encontrando distribuições semelhantes das frações de $\mathrm{HbA}$ e $\mathrm{HbA} 2$.

Lacerda \& Soto-Blanco (2006) encontraram menores valores de Hemoglobina Globular Média (HGM) em ovinos da raça Santa Inês com o genótipo HbBB quando comparados aos genótipos HbAA e $\mathrm{HbAB}$, porém não encontraram outros parâmetros hematológicos $(\mathrm{Ht}, \mathrm{Hb}, \mathrm{He}, \mathrm{VGM}$ e CHGM) com diferença, não demonstrando importância significativa na diferença dos genótipos no que se refere aos parâmetros hematológicos desta espécie ᄀ, uma vez que o parâmetro HGM não é utilizado rotineiramente para a interpretação hematológica em medicina veterinária. Da mesma forma, a presença ou ausência de $\mathrm{HbA} 2$ em cães não demonstram ser importantes no que se refere aos parâmetros hematológicos de caninos.

\section{CONCLUSÕES}

A partir dos resultados do presente trabalho, pode-se concluir que a HbA é o maior componente da hemoglobina canina, sendo detectada em todos os animais do estudo, e que uma menor parcela de animais possui uma pequena concentração de HbA2.

Não existe diferença entre as concentrações de $\mathrm{HbA}$ e HbA2 entre animais saudáveis e animais com anemias crônicas.

Agradecimentos,- À professora Ana Beatriz Monteiro Fonseca e ao Laboratório de Estatística da UFF pelo valioso auxílio dado na elaboração das análises e cálculos estatísticos indispensáveis para a conclusão deste trabalho. À CAPES pela bolsa de auxílio à pesquisa.

\section{REFERÊNCIAS}

Al-Samarrae S.H., L-Bayaati A.J. \& Al-Murrani W.K. 2010. Hemoglobin polymorphism in different animal species in Iraq. Al-Anbar J. Vet. 3(2):73-77.

Atyabi N., Rahbarghazi R. \& Araghi A. 2012. Haemoglobin typing and its variations in Iranian domestic dogs. Comp. Clin. Pathol. 21:1515-1519.

Ayres M.N.N., Birgel Júnior E.H., Rosenfeld A.M.F. \& Birgel E.H. 2005. Polimorfismo da hemoglobina de bubalinos (Bubalis bubalis) da raça Murrah criados no estado de São Paulo, Brasil. Revta Bras. Saúde Prod. Anim. 6(1):18-23.

Ayres M.C.C., Chaves Filho R.M., Rocha J.S., Gama S.M.S., Cavalcanti A.S.R., Almeida M.A.O. \& Bittencourtt T.C.B.S.C. 2009. Polimorfismo da hemoglobina de ovinos (Ovis aries L.): determinação do tipo e da frequência em animais criados no Estado da Bahia. Revta Bras. Saúde Prod. Anim. 10(3):637-644.

Ayres M.C.C., Silva M.N., Pereira A.F., Meneses I.D.S. \& Zanutto M.S. 2011. Frequências genotípicas do polimorfismo da hemoglobina de felinos 
atendidos no Hospital de Medicina Veterinária da Universidade Federal da Bahia. Revta Ciênc. Agrovet. 10(Número Especial):1-3.

Barca Junior F.A., Okano W., Thomazella E.Z., Baran M.R. \& Sturion T.T. 2008. Determinação das frequências genotípicas e alélicas do polimorfismo de hemoglobina em bovinos da raça bonsmara no norte do estado do Paraná. Arq. Ciênc. Vet. Zool. Unipar 11(1):31-34.

Egena S.S.A. \& Alao R.O. 2014. Haemoglobin polymorphism in selected farm animals: a review. Biotechnol. Anim. Husbandry 30(3):377-390.

Esharatkhah B., Safi S., Khaki Z., Khazarei N. \& Sadaghian M. 2007. Study on haemoglobin polymorphism in two breeds of Iranian sheep. J. Anim. Vet. Adv. 6(12):1426-1428.

Jain N.C. 1993. Essentials of veterinary hematology. Lea and Febiger, Philadelphia. 417p.

Kitchen H. \& Brett I. 1974. Embryonic and fetal hemoglobin in animals. Annals New York Acad. Sciences 241:653-671.

Lacerda R.M. \& Soto-Blanco B. 2006. Determinação das variantes de hemoglobina em ovinos mestiços Santa Inês. Revta Caatinga 19(4):345349.

Leoneli G.G., Imperial R.E., Marchi-Salvador D.P., Naoum P.C. \& Bonini-Domingos C.R. 2000. Hemoglobinas anormais e dificuldade diagnóstica. Revta Bras. Hematol. Hemoterapia 22(3):396-403.

Lin M., Wang Q., Zheng L., Huang Y., Lin F., Lin C.P. \& Yang L.Y. 2012. Prevalence and molecular characterization of abnormal hemoglobin in eastern Guangdong of southern China. Clin. Genetics 81:165-171.

Luz D.O., Lacerda R.M., Barreto Júnior R.A. \& Soto-Blanco B. 2010. Eritrograma e variantes de hemoglobina em caprinos da raça Canindé. Arq. Bras. Med. Vet. Zootec. 62(1):208-210.

Naik S.N., Anderson D.E., Jardine J.H. \& Clifford D.H. 1971. Glucose-6-phosphate dehydrogenase deficiency, haptoglobin and hemoglobin variants in dogs. Anim. Blood Groups Biochem. Genetics 2:89-94.

Naoum P.C. 1987. Coleta de sangue e preparação de hemolisado, p.173174. In: Naoum P.C. (Ed.), Diagnóstico das Hemoglobinopatias. Sarvier, São Paulo.
Pal S.K. \& Mummed Y.Y. 2014. Investigation of haemoglobin polymorphism in Ogaden cattle. Vet. World 7(4):229-233.

Ramos P.R.R., Bortolozzi J., Kohayagawa A. \& Caminhas M.M.T. 1997. Variação polimórfica de hemoglobinas em búfalos (Bubalus bubalis). Braz. J. Vet. Res. Anim. Sci. 34(2):85-89.

Regis G.B. 2002. Tipos de hemoglobina de caprinos das raças Saanen, Toggenburg e sem raça definida criados na região Semi-Árida do Estado da Bahia: influência de parâmetros hematológicos e do parasitismo gastrintestinal. 58p. Dissertação de Mestrado, Universidade Federal da Bahia, Salvador. 58p.

Rego F.C.A., Barca Junior F.A., Cunha Filho L.F.C., Okano W., Silva L.C., Ludovico A., Trapp S.M., Santana E. \& Tanaka N.M. 2011. Polimorfismo de hemoglobina em rebanho de ovinos Hampshire Down. Semina, Ciênc. Agrárias 32(1):261-266.

Seal U.S. 1969. Carnivora systematics: a study of hemoglobins. Comp. Biochem. Physiol. 31(5):799-811.

Seixas F.A.V., Silva C.D., Tominaga J., Ferro O.C. \& Nilson L.G. 2008. Incidence of hemoglobinopathies in Northwest Paraná, Brazil. Revta Bras. Hematol. Hemoterapia 30(4):287-291.

Srivorakun H., Fucharoen G., Changtrakul Y., Komwilaisak P. \& Fucharoen S. 2011. Thalassemia and hemoglobinopathies in Southeast Asian newborns: diagnostic assessment using capillary electrophoresis system. Clin. Biochem. 44:406-411.

Tanabe Y., Omi T. \& Ôta K. 1978. Genetic variants of hemoglobin in canine erythrocytes. Anim. Blood Groups Biochem. Genetics 9:79-83.

Wagner S.C., Silvestri M.C., Bittar C.M., Friedrisch J.R. \& Silla L.M.R. 2005. Prevalência de talassemias e hemoglobinas variantes em pacientes com anemia não ferropênica. Revta Bras. Hematol. Hemoterapia 27(1):3742.

Yakubu A., Abimiku H.K., Musa-Azara I.S., Barde R.E. \& Raji A.O. 2014. Preliminary investigation of haemoglobin polymorphism and association with morphometric traits in West African Dwarf goats in north central Nigeria. Mljekarstvo 64(1):57-63. 